\title{
Utopia 89 - Un projet théâtral et scientifique sur la manifestation du 4 novembre 1989 à Berlin-Est
}

\author{
Caroline Moine ${ }^{1}$, Guillaume Mouralis ${ }^{2}$, Laure de Verdalle ${ }^{3}$
}

Le projet " Utopia $\underline{89} \|^{4}$ est né d'une rencontre entre les auteur.es de cet article, spécialistes de la RDA et des transformations qui ont accompagné sa disparition ${ }^{5}$, et Frédéric Barriera, qui a écrit et mis en scène une pièce de théâtre inspirée d'un événement précis de l'automne 1989. Le 4 novembre 1989, une manifestation d'une ampleur sans précédent a lieu à Berlin-Est. Initiée par l'opposition et autorisée à contrecœur par le pouvoir, elle s'achève par un meeting d'environ trois heures sur l'Alexanderplatz où 25 personnalités du monde artistique, intellectuel et politique de RDA prennent la parole pour réclamer une démocratisation grâce à des réformes politiques en profondeur du pays et un socialisme rénové. Au cours du meeting, six artistes interprètent des chansons volontiers impertinentes. Rétrospectivement, la place de ce moment dans l'économie de la "révolution pacifique » en RDA apparaît mal assurée, compte tenu des événements qui l'ont suivi (chute du mur cinq jours plus tard et unification allemande à marche forcée dans les mois suivants). Son sens et sa portée restent controversés et tributaires du point de vue et de la position des protagonistes. II mérite donc d'être interrogé et resitué dans son contexte "révolutionnaire ».

Nous avons fait le pari que les approches artistique et scientifique de l'événement pouvaient se féconder mutuellement. Cette coopération a débouché sur un projet articulant plusieurs volets : outre la pièce de théâtre, dont le texte a fait l'objet de discussions collectives, nous avons conduit une enquête approfondie sur le 4 novembre 1989, à partir d'archives et d'entretiens ${ }^{7}$. Un colloque international, «Die Straße ist die Tribüne des Volkes - Ansichten zum 4. November 1989 », organisé à Berlin les 30 et 31 octobre $2019^{8}$, nous a permis de présenter les premiers résultats d'une enquête

\section{La pièce de théâtre Utopia 89 / Nous sommes le peuple}

Des discours, souvent ciselés et incisifs, du 4 novembre 1989, F. Barriera a fait la matière d'une pièce francoallemande originale. Interprétée par deux acteurs, Jürgen Genuit et Amandine Thiriet, elle a été jouée à 17 reprises à Paris et à Berlin d'octobre à décembre 2019. En voici, succinctement, l'argument : «Un aède raconte l'histoire d'un petit pays disparu qui, dans un dernier sursaut, a tenté de concrétiser une utopie. Une autre voix, féminine, invite le peuple à rêver d'un autre monde possible (...) " .
Puis le spectateur assiste à une répétition durant laquelle deux comédiens, un homme et une femme, attendent en vain le célèbre dramaturge est-allemand Heiner Müller, censé mettre en scène la pièce où ils jouent. Livrés à euxmêmes, ils lisent les discours prononcés le 4 novembre 1989 sur l'Alexanderplatz, et « se demandent quoi faire théâtralement de ce matériau historique. Que voulaient donc ces orateurs ? Et la population ? Le pouvoir ? Comment dire les textes de Christa Wolf ou Marianne Birthler et leur donner une résonance aujourd'hui ? Comment faire entendre cette matière, faite d'utopie, trente ans après la chute du mur ? ${ }^{6}$ »

\footnotetext{
Maîtresse de conférences en histoire contemporaine, Université Paris-Saclay, UVSQ/CHCSC, chercheuse associée au Centre Marc Bloch (Berlin).

2 Chercheur en science politique et histoire, CNRS/Centre Marc Bloch (Berlin).

Chargée de recherche en sociologie, CNRS/Centre Marc Bloch (Berlin).

Une présentation détaillée du projet et de ses différents volets est disponible sur le site : https://nslp.hypotheses.org/ [consulté le 24/03/2020].

5 Nous avons respectivement travaillé sur le cinéma est-allemand dans la guerre froide (Moine 2014), les procès des dirigeants est-allemands après la réunification (Mouralis 2008), et les transformations du monde théâtral est-allemand après 1990 (Verdalle 2006).

6 F. Barriera, « Présentation de la pièce » sur le site : https://nslp.hypotheses.org/la-piece [consulté le 24/03/2020].

7 Nous avons consulté, à Berlin, les fonds de la Robert Havemann Gesellschaft (archives de l'opposition en RDA), du Bundesarchiv de Berlin-Lichterfelde, de l'Akademie der Künste, du Deutsches Theater et du Deutsches Rundfunkarchiv. A cela s'ajoute une dizaine d'entretiens menés par les chercheur.e.s et le metteur en scène avec des organisatrices de la manifestation (Jutta Wachowiak, Jutta Seidel), des oratrices et orateurs (Marianne Birthler, Joachim Tschirner) et de " simples » manifestantes (Christine Boyde, Silke Haase).

8 Ce colloque ("La rue est la tribune du peuple - regards sur le 4 novembre 1989 ») a été organisé conjointement par le Centre Marc Bloch et l'Institut français de Berlin, avec le soutien de la Bundeszentrale für politische Bildung, de l'Office franco-allemand pour la jeunesse et de la Rosa-Luxemburg-Stiftung. Le programme détaillé, en allemand, de cette conférence est disponible à cette adresse : https://cmb.hu-berlin.de/fr/agenda/evenement/utopia89-die-strasse-ist-die-tribuene-des-volkes/ [consulté le 24/03/2020].
}

Ә Open Access. $\odot 2020$ Caroline Moine et al., published by Sciendo. (cc) BY-NC-ND This work is licensed under the Creative Commons Attribution NonCommercial-NoDerivatives 4.0 License. 
qui portait sur la genèse, le déroulement de la manifestation et sa construction en événement. A travers ses différents panels, le colloque a aussi traité des dynamiques révolutionnaires en RDA et en Europe de l'Est, et abordé les mémoires du 4 novembre. Comme l'ont montré plusieurs communications $^{9}$, « la révolution pacifique » doit beaucoup aux profonds changements intervenus dans le Bloc de l'Est depuis 1988 au moins (table ronde et élections libres en Pologne), des changements accélérés par la crise des réfugiés est-allemands au cours de l'été 1989. En RDA, la crise de l'automne 1989 reflète également l'incapacité des marxistes critiques au sein du parti à susciter des réformes.

Le colloque a été précédé, le 29 octobre, par la première représentation de la pièce Utopia 89 . Nous sommes le peuple à l'Institut français de Berlin et s'est accompagné de l'inauguration d'une exposition, accueillie jusqu'en janvier 2020 par le Centre Marc Bloch, que nous avons conçue comme une forme de restitution de notre travail d'enquête ${ }^{10}$. Une table ronde, intégrée au programme du colloque, a également rassemblé différent.es protagonistes (Jutta Seidel, membre fondatrice du mouvement citoyen Neues Forum, Tina Krone archiviste à la Robert-Havemann-Gesellschaft, Silke Haase, artiste berlinoise originaire de RDA, et Andreas Kämper, photographe présent sur l'Alexanderplatz le 4 novembre 1989), qui ont confronté leurs souvenirs de l'événement. L'ensemble de ce projet constitue donc une véritable entreprise collective qui a eu la (rare) capacité de réunir des chercheur.es, des artistes et des témoins.

Depuis trente ans en Allemagne, des débats récurrents ont lieu entre historien.nes, sur le sens et la portée de la « révolution pacifique ${ }^{11}$, au sein desquels le 4 novembre 1989 occupe presque toujours une place problématique (Braun / Schäbitz 2016 ; Kowalczuk 2009 ; Pollack 2000 ; Rübesame 2010 ; Süß 2012 : 385-413 ; Bauer 2019). Cet événement atypique vient perturber les "grands " récits historiques, en particulier celui d'une réunification allemande inéluctable. Le projet Utopia 89 a permis de reconsidérer cet événement, sa genèse et sa construction, en l'appréhendant sans idées préconçues et en portant une attention particulière aux différent.es actrices et acteurs impliqués et à leurs interactions dynamiques dans une conjoncture politique particulièrement « fluide ${ }^{12}$. Loin de pouvoir restituer ici toute la richesse de ce projet et de ses différents volets, nous allons nous focaliser sur les éléments issus de notre enquête empirique en évoquant successivement les négociations qui ont entouré l'organisation de la manifestation, son déroulement et enfin sa médiatisation.

9 Paulina Gulińska-Jurgiel (Martin-Luther-Universität Halle-Wittenberg), « Ein Kind vieler Eltern ? Aktivisten, Vermittler und Beobachter - Das Jahr 1989 in Polen und seine (ausgewählten) Akteure »; Detlef Pollack (Universität Bremen), " Haben die Oppositionsgruppierungen die friedliche Revolution herbeigeführt ? Beobachtungen zum Umbruch in der DDR »; Sonia Combe (Centre Marc Bloch) : « Das Scheitern der kritischen Marxisten in der DDR $»$;
DDR $»$.

10 L'exposition "Utopia'89 / Spuren einer Utopie" [" Utopia'89 / Traces d'une utopie "] présente plusieurs documents d'archives et de nombreuses photos prises lors de la man festation. Constituée de treize panneaux, elle est désormais disponible pour un nouvel accrochage, les demandes peuvent être adressées aux auteur.e.s de cet article.

11 Le dernier en date est un débat plutôt vif qui a opposé dans la presse, au cours de l'été 2019, plusieurs spécialistes de 1989, en particulier Detlev Pollack et Ilko-Sascha Kowalczuk. 12 Au sens où l'entend Michel Dobry (1986 : 140-150 ; 1995, consulté le 26/03/2020).

\section{Les préparatifs du 4 novembre 1989}

\section{La genèse d'un événement et son contexte}

Le 15 octobre 1989, le Deutsches Theater à Berlin accueille un grand rassemblement de gens de théâtre venus de toute la RDA. Lors de cette réunion, à laquelle assistent environ 700 personnes, la décision est prise d'organiser une manifestation à Berlin.

Pour comprendre la genèse de cette décision, il faut la réinscrire dans une chronologie un peu plus large. En effet, dès septembre 1989, différentes associations artistiques (Künstlerverbände) adoptent des positions appelant à des réformes politiques. Dans le même temps, une forme d'opposition se structure : Neues Forum est fondé les 9 et 10 septembre 1989, de nombreux artistes sympathisent avec ce mouvement citoyen et relaient ses prises de position. Les 7 et 8 octobre, les célébrations du $40^{\mathrm{e}}$ anniversaire de la RDA sont marquées par des violences policières qui soulèvent de nombreuses protestations. Le 9 octobre, lors de la prière du lundi, 70000 manifestants sont présents à Leipzig. Les forces de police n'interviennent pas et le régime montre des signes d'indécision, quant à la manière de gérer un mouvement de contestation qui prend chaque jour de l'ampleur et qui se manifeste aussi à travers la fuite d'un nombre croissant de citoyens est-allemands, via la Tchécoslovaquie et la Hongrie.

Le 14 octobre, Jutta Seidel, médecin de Berlin-Est et membre fondatrice de Neues Forum, écrit à Jutta Wachowiak, actrice au Deutsches Theater, qui est aussi sa voisine. Le message qu'elle dépose dans sa boite aux lettres suggère la tenue d'une manifestation à Berlin. Neues Forum, qui ne dispose encore d'aucune existence légale, peut difficilement l'organiser, du moins de manière officielle. Or c'est bien le principe d'une manifestation au grand jour, pour laquelle une demande d'autorisation pourrait être formulée, qui fait son chemin parmi les membres de Neues Forum et chez les artistes qui leur sont proches. Le lendemain, J. Wachowiak relaie cette proposition lors du rassemblement qui a lieu au Deutsches Theater et reçoit le soutien enthousiaste de ses collègues. Plus tôt dans la journée, l'avocat Gregor Gysi, proche des milieux artistiques, avait déjà plaidé pour l'adoption de formes de protestation adossées à des moyens légaux.

\section{Une manifestation « négociée »}

Un groupe de gens de théâtre berlinois, l'Initiativgruppe 4.11, appartenant à différentes générations et plus ou moins proches des mouvements citoyens, se met en place pour organiser concrètement la manifestation.

Le 17 octobre, Wolfgang Holz (comédien, représentant syndical au Berliner Ensemble et membre du groupe d'initiative) dépose une demande d'autorisation auprès de la police de Berlin-Est, pour la tenue d'une manifestation défendant les articles 27 et 28 de la constitution de la RDA (liberté de réunion et d'opinion, liberté de la presse et des médias). 
Jusqu'à la fin du mois d'octobre, cette demande et les détails matériels de la manifestation (date, parcours, liste des intervenants au meeting qui viendra clore le défilé) seront discutés dans plusieurs lieux et en présence de différents interlocuteurs : les membres du groupe d'initiative ; la police de Berlin-Est ; le comité central du Parti socialiste unifié d'Allemagne (SED) ; la direction berlinoise du SED ; le ministère de la Culture ; la Stasi, qui suit de près les préparatifs ; les présidents des différentes associations d'artistes de la RDA ; les intendants des théâtres berlinois ; et les membres des mouvements citoyens, dont Neues Forum, qui ne participent pas officiellement aux discussions, mais qui y sont indirectement associés par le biais de leurs relations avec certaines et certains artistes ou via leurs prises de positions dans d'autres arènes.

L'information concernant l'organisation de la manifestation berlinoise se diffuse très rapidement, dans les réseaux artistiques et les mouvements citoyens. Le régime semble alors pris de vitesse. Le 20 octobre, Kurt Hager (membre du comité central du SED où il est en charge des questions de culture) s'adresse à Egon Krenz, sur le point de remplacer Erich Honecker, et lui recommande de chercher à contrôler la manifestation, plutôt que de l'interdire, pour en faire l'incarnation de la Wende («tournant ») promue par le régime.

Des négociations ont lieu entre l'Initiativgruppe 4.11 et les autorités, notamment policières. Elles concernent d'abord le parcours de la manifestation et le lieu du meeting final, qui sera déplacé de la Platz der Akademie, jugée trop proche du mur, vers l'Alexanderplatz. Elles portent ensuite sur le choix des orateurs, qui prendront la parole à la tribune. Des représentants officiels sont invités à s'exprimer. Les organisatrices et organisateurs, dans leur volonté de réformer et démocratiser la RDA, favorisent un large spectre d'opinions et de positions. La place particulière que les artistes et gens de théâtre occupent en RDA rend possible ce rôle de médiateur. II faut aussi noter que, dans le contexte de l'automne 1989, la composition, en apparence hétérogène, de la liste des orateurs du 4 novembre fait écho à d'autres événements et rencontres. Ainsi, le 24 octobre, une table ronde « La RDA - comme je la rêve " fait dialoguer, dans la "Maison des jeunes talents » à Berlin, Markus Wolf avec Bärbel Bohley et Jens Reich (tous deux membres fondateurs de Neues Forum). A l'inverse, Wolf Biermann, déchu de sa citoyenneté est-allemande en 1976, invité par Bärbel Bohley à participer à la manifestation, ne figure finalement pas sur la liste des orateurs, pour éviter toute provocation.

\section{Le 4 novembre comme expérimentation politique}

\section{Déroulement et itinéraire de la manifestation}

Entre 200000 et 400000 personnes défilent à Berlin-Est le 4 novembre 1989, ce qui représente une participation record, inédite dans la capitale est-allemande depuis la révolte de juin 1953. Le dispositif négocié entre les autorités et l'opposition comprend deux volets : la manifestation proprement dite, commençant comme annoncé à 10 heures du matin, se déroule de l'Agence de presse est-allemande (ADN) jusqu'à l'Alexanderplatz en suivant un parcours prédéfini. Elle est suivie d'un meeting de trois heures environ, au cours duquel chacun des 25 intervenants parle pendant quelques minutes sur une tribune improvisée. Ce meeting assez théâtral dans sa conception, modéré par le scénographe Henning Schaller, est ponctué de chansons mêlant humour, impertinence politique et mélancolie.

Les forces de sécurité, quoique présentes et visibles, restent à distance, si bien que les manifestants peuvent s'approcher des bâtiments officiels et des centres du pouvoir tels que le Palais de la République (siège de la Chambre du Peuple) ou le Conseil d'État. La police laisse les militants coller des affiches sur les murs de ces bâtiments. L'une d'elles, sur un mur du Conseil d'État, proclame : " Pluralismus statt Parteimonarchie! " [Le pluralisme plutôt que la monarchie de parti ! ]. La présence policière importante vise essentiellement à empêcher la manifestation de s'approcher du mur de Berlin, une éventualité envisagée avec inquiétude par les autorités ; en outre, la Stasi, à l'insu des manifestants, a dissimulé des « volontaires » à l'intérieur du Palais de la République, prêts à intervenir en cas d'incident.

\section{L'éclosion d'une culture protestataire}

La principale revendication des organisateurs de la manifestation concerne l'application des articles 27 et 28 de la constitution de la RDA (liberté de réunion et d'opinion, liberté de la presse et des médias). En outre, de nombreuses banderoles réclament la reconnaissance officielle des « mouvements de citoyens " créés depuis septembre 1989, tels que Neues Forum, et l'organisation d'élections libres. La plupart des slogans, des banderoles et des affiches appellent à la démocratisation de la RDA sans nécessairement mettre en cause les fondements socialistes de l'État.

Trois aspects du régime autoritaire est-allemand sont tout particulièrement visés. Banderoles et affiches dénoncent tout d'abord la prétention du Parti socialiste unifié d'Allemagne (SED) à diriger seul le pays. Le nom même du nouveau secrétaire général du parti, Egon Krenz, qui a succédé à Erich Honecker le 17 octobre, inspire de nombreux jeux de mots. Les manifestants prennent également pour cible l'exercice politique de la justice et la toute-puissance des forces de sécurité, la Stasi étant particulièrement en ligne de mire. Enfin, au cours de la journée, les participants dénoncent vivement les privilèges, les abus de pouvoir et la corruption des élites du parti-État. Toutefois, la démocratisation des institutions politiques n'est pas la seule préoccupation des manifestants. Les questions écologiques, centrales pour les cercles d'opposition en RDA depuis le début des années 1980, sont présentes. Moins visibles, les mouvements libertaires, anarchistes et « antifa » participent activement à la manifestation : dénonçant toute forme d'autorité, ils exigent un service civil se substituant au service militaire et appellent à combattre les néo-nazis en RDA (Kloft et al. 1989). 
Ce qui frappe dans cette éclosion libératrice de revendications diverses et de slogans audacieux, c'est l'absence (ou du moins la marginalité) de certaines questions : les bouleversements politiques dans les autres pays d'Europe de l'Est sont étonnamment peu abordés, mis à part quelques mots de solidarité envers Vaclav Havel écrits sur un calicot en tête de cortège. Surtout, la question nationale allemande est presque entièrement absente, à l'exception d'un carton appelant à la « réunification » furtivement saisi par la caméra de la télévision est-allemande - une vision étrange, presque anachronique dans le contexte de cette manifestation.

\section{Profil professionnel et politique des 25 orateurs et des 6 chanteurs}

Même si les intellectuels et les artistes de Berlin-Est forment la majorité des oratrices et orateurs qui se succèdent, trois heures durant, à la tribune, ce groupe est beaucoup plus hétérogène en termes de profession, de milieu social et politique que ne le laissent supposer les comptes rendus rétrospectifs du 4 novembre.

Parmi les 25 orateurs, on compte cinq femmes - une écrivaine, une représentante des mouvements citoyens et trois comédiennes, dont Steffie Spira qui clôt le meeting en convoquant Brecht contre les hiérarques du régime. Seize des orateurs travaillent dans les domaines artistique ou littéraire, dont dix au théâtre (huit actrices et acteurs, un scénographe et un dramaturge, en l'occurrence Heiner Müller). Cinq représentants des mouvements de citoyens prennent la parole : Jens Reich et Henning Schaller de Neues Forum Marianne Birthler du « Groupe de contact téléphonique de Berlin » chargé de faire la lumière sur les violences policières des 7 et 8 octobre ; les pasteurs Friedrich Schorlemmer et Konrad Elmer, respectivement cofondateurs du mouvement Demokratischer Aufbruch et du Parti social-démocrate de RDA.

À l'autre extrémité du spectre politique, cinq membres du SED s'expriment, dont un membre de la direction politique renouvelée, Günter Schabowski et l'ancien chef du contrespionnage, Markus Wolf. A ces derniers, on peut ajouter les membres « ordinaires » du parti - mais parfois critiques à son égard - tels que le professeur Lothar Bisky, l'avocat Gregor Gysi ou encore l'écrivaine Christa Wolf. Ajoutons qu'un autre représentant du pouvoir, Manfred Gerlach, président du LDPD (un des partis du « Bloc » inféodés au SED) et viceprésident du Conseil d'État, prend également la parole.

Ce 4 novembre, les " nouveaux " représentants du pouvoir, Schabowski en tête, sont copieusement sifflés et hués par la foule, malgré leurs promesses de réformes. Ceci montre à quelle vitesse le capital politique " de type soviétique " se trouve dévalué (Bourdieu 1994 ${ }^{13}$ ). De ce point de vue, on peut constater la profondeur de la crise : les détenteurs d'un capital exclusivement politique - la génération des fondateurs de la RDA incarnée par Honecker - ont été remerciés le 18 octobre. Les représentants de la génération suivante, tels

13 Intervention à Berlin-Est le 25 octobre. que Schabowski, qui disposent de ressources non seulement politiques mais aussi académiques et qui se sont tardivement présentés comme réformateurs, ne sont eux-mêmes plus audibles. La crise du capital politique de type soviétique a successivement balayé les deux groupes. Seul Gregor Gysi sort plutôt indemne du 4 novembre, car il se situe alors en dehors (ou seulement à la périphérie) du champ de pouvoir de la RDA.

\section{La médiatisation du 4 novembre, un tournant de la révolution pacifique?}

\section{Les médias d'information face à la montée des contestations}

On l'a vu, l'une des principales revendications de la manifestation du 4 novembre 1989 concerne la liberté de réunion et d'expression. II est cependant remarquable que, parmi les personnes qui prennent la parole ce jour-là, seules deux représentent le milieu professionnel des médias : le documentariste des studios de la DEFA, Joachim Tschirner, et Lothar Bisky, recteur de l'École de cinéma et de télévision Konrad Wolf de Potsdam-Babelsberg ${ }^{14}$. Les médias n'en demeurent pas moins au cœur de plusieurs des discours tenus, et ils ont contribué, parmi d'autres facteurs bien sûr, mais de manière essentielle, à en faire un événement historique.

Certes, dans les semaines précédant le 4 novembre, la situation a déjà beaucoup évolué. Lorsque Aram Radomski et Siegbert Schefke filment à Leipzig la manifestation du lundi 9 octobre au soir, se joue sans aucun doute le moment de rupture essentiel dans la médiatisation des contestations, représentant une brèche décisive dans la censure imposée par Berlin-Est (Schefke / Martell 2019). Les images, aussitôt envoyées en RFA et diffusées le lendemain soir sur la principale chaîne ouest-allemande, ont pu en effet être vues par les téléspectateurs des deux Allemagnes. De leurs côtés, les étudiants de l'École de cinéma, puis certains documentaristes de la DEFA commencent à filmer ce qui se déroule sous leurs yeux. Puis, le 24 octobre, la table ronde, "La RDA comme je la rêve " à laquelle participent des représentants du gouvernement et de l'opposition, comme une répétition avant le 4 novembre, est non seulement retransmise en direct à la télévision est-allemande mais diffusée également le lendemain en RFA.

\section{Quelles images de la manifestation?}

La décision de retransmettre en direct la manifestation du 4 novembre, et plus précisément le meeting, intervient donc dans un contexte de bouleversement du rôle des médias qui s'ouvrent de plus en plus aux prises de parole publiques et critiques. II reste cependant difficile de retracer précisément le processus qui a conduit à cette décision qui, semble-t-il, est restée incertaine jusqu'à la dernière minute. La portée de ces images est forte. En effet, elles montrent, sans coupure ni commentaire, à des millions de téléspectateurs, comment

14 Le critique de théâtre Klaus Baschleben ne représentait, lui, aucune institution. 
une foule immense, réunie non plus de nuit comme à Leipzig mais en plein jour, hue et moque les représentants du PartiÉtat - dans un processus proprement révolutionnaire.

D'autres images nous permettent de mieux saisir l'atmosphère tout à la fois joyeuse et anxieuse de cette journée, au-delà du seul meeting, et dont les participants sont largement conscients de vivre un événement historique ${ }^{15}$. Les photographies et les films de la Stasi ainsi que les commentaires qui les accompagnent témoignent tout à la fois des ultimes tentatives de contrôle et aussi d'un grand désarroi de la part de ses membres ${ }^{16}$. Mais ce sont surtout de très nombreux photographes, cameramen, journalistes et anonymes, allemands (de l'Est comme de l'Ouest) ou étrangers, qui saisissent, eux, de manière totalement transparente, la grande diversité des manifestants, hommes, femmes, jeunes et moins jeunes, seuls, en famille ou en groupe. Du côté est-allemand, le réalisateur Thomas Heise filme au plus près les oratrices et orateurs, avant le meeting puis, directement, à la tribune ${ }^{17}$ Le photographe Andreas Kämper, qui a tenu un journal photographique d'octobre 1989 à novembre 1990, saisit plusieurs moments du défilé et du meeting, y compris dans leurs marges - ce que la télévision ne montre pas ${ }^{18}$.

Dans les informations télévisées du soir, le compte rendu de la journée, centré sur les discours, est suivi d'une déclaration des membres du SED de la télévision est-allemande présentant leurs excuses pour leur responsabilité dans la crise que traverse la RDA. Le 4 novembre constitue ainsi un moment important de mise en scène de la télévision est-allemande par elle-même mais aussi de son autocritique, afin de regagner la confiance des téléspectateurs Plus largement, les journaux comme la télévision parlent de Berlin-Est mais évoquent également les autres manifestations qui se sont déroulées ce même jour dans le reste de la RDA, ainsi que de la poursuite des départs massifs vers la RFA. Les médias ont donc contribué à la construction de la manifestation du 4 novembre comme événement historique de la "révolution pacifique ", mais, dans le même temps, l'ont immédiatement resitué dans le flux des autres événements.

\section{Conclusion}

Le projet Utopia 89 a constitué une expérience originale d'association étroite entre une démarche de recherche et un processus de création artistique. Les analyses que nous avons conduites à partir des matériaux d'archives et des entretiens sont venues éclairer le travail de l'auteur et metteur en scène, Frédéric Barierra. II a été intéressant de

\footnotetext{
15 Voir les témoignages de Tina Krone, Jutta Seidel et Andreas Kämper lors de la table ronde du colloque.

16 Voir le chapitre 12 du DVD Fremdbilder (2006), de la Bundeszentrale für politische Bildung : https://www.bpb.de/mediathek/595/wende [consulté le 26/03/2020].

17 Le cinéaste a utilisé quelques minutes de ces images au début de son film documentaire Material (Heise 2009: 2h46).

18 Andreas Kämper a déposé l'ensemble de ses photographies concernant la révolution pacifique (plus de 4000 ) aux archives de la Robert-Havemann-Gesellschaft. L fonds est en partie visible en ligne : https://www.havemann-gesellschaft.de/archiv-der-ddropposition/bildarchiv/bestaende-einzelner-fotografen/bestand-kaemper-andreas/ [consulté le 26/03/2020].
}

\section{Mise en musée et commémorations du 4 novembre 1989}

« La profusion de banderoles que l'on peut voir ici est vraiment frappante et ce serait très bien, je trouve, si nous pouvions collecter ces banderoles et les utiliser pour une sorte d'exposition artistique d'un nouveau genre " lance le modérateur Henning Schaller à la fin du meeting. Ces paroles posent la question des suites à donner à la manifestation et à cette mobilisation collective si inventive et novatrice pour la culture politique est-allemande.

Dans les semaines et les mois qui suivent le 4 novembre, plusieurs tentatives de muséalisation répondent à cette attente. Lotte Thaa (FU Berlin) a présenté, lors du colloque Utopia 89, les expositions autour du 4 novembre et de ses banderoles, qui ont été organisées à Berlin-Ouest, Berlin-Est et Bonn entre 1989 et 1990. Elle a souligné les tensions qui sont rapidement apparues sur le sens à donner à la manifestation (tentative de renverser ou de sauver le SED ?), y compris entre les organisateurs du 4 novembre.

Suit le temps des commémorations. Thomas Flierl (ancien sénateur à la culture de Berlin) est ainsi revenu sur celles du 4 novembre 1999 qu'il avait coorganisées. Dix ans après, les images de la retransmission télévisée du meeting sont projetées sur l'Alexanderplatz; une exposition ainsi que des débats réunissent des orateurs et participants de 1989. Tout l'espace de la célèbre place se trouve ainsi à nouveau investi des images, sons et expériences de la manifestation, dans un contexte politique nouveau, à l'échelle berlinoise et nationale, proposant d'interroger à travers la mémoire du 4 novembre dix années d'unité allemande.

Trente ans après 1989, la date du 4 novembre semble sortir d'un relatif oubli et s'inscrire à nouveau dans la mémoire collective de la "révolution pacifique ». Ainsi, la semaine des commémorations officielles de 2019 s'est ouverte précisément le lundi 4 novembre, sur l'Alexanderplatz. Un collectif d'artistes berlinois, Panzerkreuzer Rotkäppchen (PKRK), dont deux membres, Simon Strick et Anna Stiede, sont venus présenter leur projet lors du colloque, s'est emparé - sur un mode curieusement grotesque - des discours du meeting pour en faire un spectacle mêlant musique, danse, et prises de paroles d'activistes de mobilisations actuelles (Fridays for Future, etc.). Proposant une réflexion sur les échos actuels des discours, attentes et espoirs de 1989, leur création interroge le rôle des émotions en politique - émotions qui furent si fortes ce 4 novembre 1989.

confronter, dans des échanges parfois passionnés, nos regards d'historien.nes et de sociologue à celui d'un artiste, cherchant non pas à recréer l'événement, mais à questionner la pluralité des mémoires et des points de vue auquel il peut donner lieu.

La manifestation du 4 novembre 1989 se prête particulièrement bien à ce type de réflexions. Le processus qui conduit à son organisation est, nous l'avons vu, jalonné de négociations et de formes de compromis, qui vont, par 
la suite, nourrir des mémoires parfois contradictoires et fragmentées de l'événement. Certains y voient une ultime tentative du régime de reprendre la main sur une situation de crise, quand d'autres célèbrent le rôle joué par les mouvements d'opposition et la libération de la parole qui transparaît des slogans brandis par les manifestants et des discours de certains orateurs. Aujourd'hui, toutefois, la question n'est peut-être pas tant de savoir si le 4 novembre 1989 vient conforter la Wende qu'Egon Krenz cherchait à mettre en avant, ou précipiter la fin du régime du SED. En effet, selon les termes utilisés par le journaliste allemand Patrick Bauer, dans l'ouvrage qu'il a récemment consacré à cette manifestation, le 4 novembre 1989 est un « jour entre les temps » ("Tag zwischen den Zeiten », Bauer, 2019, p. 16), qui s'inscrit dans la dynamique de la "révolution pacifique » de l'automne 1989, mais qui se trouve aussi brutalement éclipsé par l'ouverture du mur de Berlin, cinq jours plus tard, le 9 novembre. Ce mur, si peu présent dans les discours prononcés sur l'Alexanderplatz le 4 novembre ${ }^{19}$, devient soudain le centre de l'attention mondiale et, de manière durable, un symbole des bouleversements de 1989

Notre projet de recherche, comme le geste artistique de Frédéric Barierra, constitue une tentative de déplacer un peu la focale pour ouvrir d'autres perspectives sur cette période. La manifestation, sa genèse, son déroulement, les espoirs qu'elle suscite, tracent, au moins pour certains des acteurs de l'époque, une voie possible de démocratisation de la RDA, cette voie qui sera très vite interrompue et redéfinie par la chute du mur d'abord puis par les élections de mars 1990. Porter notre regard sur cet événement singulier, comprendre les positions, souvent complexes, de ceux qui contribuent à le faire exister, permet de saisir les dynamiques contradictoires qui caractérisent les derniers mois de la RDA.

19 Ainsi, la question centrale posée par les oratrices et orateurs sur l'Alexanderplatz fut selon des modulations variées - celle de la démocratisation de leur « patrie » socialiste : le 4 novembre 1989, la question nationale n'a pratiquement joué aucun rôle.

\section{Bibliographie}

Bauer, Patrick (2019), Der 4. November 1989 und seine Geschichte. Der Traum ist aus. Aber wir werden alles geben, dass er Wirklichkeit wird, Berlin, Rowohlt.

Bourdieu, Pierre (1994), « La variante 'soviétique' et le capital politique », in Id., Raisons pratiques, Paris, Seuil, 31-35.

Braun, Jutta / Schäbitz, Michael (2016), Von der Bühne auf die Straße. Theater und friedliche Revolution in der DDR, Berlin, Vorwerk 8.

Dobry, Michel (1995), « Les causalités de l'improbable et du probable : Notes à propos des manifestations de 1989 en Europe centrale et orientale $॥$, in Culture \& Conflits, 17, http://journals. openedition.org/conflits/322, 1-17.

Dobry, Michel (1986), Sociologie des crises politiques. La dynamique des mobilisations multisectorielles, Paris, Presses de la FNSP.

Kloft, Edith / Rau-Häring, Nelly (1989), « Ein Vorschlag für den 1. Mai: Die Führung zieht am Volk vorbei. » : Die Demonstration vom 4. November 1989 in Berlin (Ost) ; 22. Nov. 1989 - 14. Jan. 1990, eine Ausstellung des Werkbund-Archivs, Museum der Alltagskultur des 20. Jahrhunderts, Berlin (West), Werkbund-Archiv.

Heise, Thomas (2009), Material, Leipzig, Ma.Ja.De. Filmproduktion $\mathrm{GmbH}$.
Kowalczuk, Ilko-Sascha (2009), Endspiel. Die Revolution von 1989 in $\operatorname{der} D D R$, München, Beck.

Moine, Caroline (2014), Cinéma et guerre froide. Histoire du festival de films documentaires de Leipzig (1955-1990), Paris, Publications de la Sorbonne.

Mouralis, Guillaume (2008), Une épuration allemande. La RDA en procès 1949-2004, Paris, Fayard.

Pollack, Detlev (2000), Politischer Protest: politisch alternative Gruppen in der DDR, Opladen, Leske + Budrich.

Schefke, Siegbert / Martell, Maren (2019), Als die Angst die Seite wechselte - Die Macht der verbotenen Bilder, Berlin, Transit Verlag.

Rübesame, Hans (Hg.) (2010), Antrag auf Demonstration. Die Protestversammlung im Deutschen Theater am 15. Oktober 1989, Berlin, Links.

Süß, Walter (2012), Staatssicherheit am Ende. Warum es den Mächtigen nicht gelang, 1989 eine Revolution zu verhindern, Berlin, Links.

Verdalle, Laure de (2006), Le théâtre en transition. De la RDA aux nouveaux Länder, Paris, Éditions de la Maison des sciences de l'homme. 